\title{
Una propuesta analítica para el estudio de las comunidades académicas en el contexto de la institución universitaria
}

\section{An analytical study proposal of academic communities in the context of the university}

\author{
Elma Barahona Henry ${ }^{7 *}$ \\ elma.barahona@gmail.com
}

\section{Resumen}

El objetivo de este trabajo es presentar una estructura analítica para el estudio de la comunidad académica en el contexto de la institución universitaria. Articula dos perspectivas, una normativa en donde se definen a partir de la revisión teórica y empírica, los componentes estructurales de una comunidad como el trabajo académico, la libertad académica, el ethos académico y la cultura académica. Desde una perspectiva interpretativa interesa analizar cómo los integrantes de una comunidad construyen su comprensión sobre los componentes estructurales definidos y la distancia existente entre la norma y la práctica. Finalmente, a partir de la articulación de los dos enfoques, se busca tener una compresión integral de cómo se configuran los componentes estructurales en una comunidad académica que está inserta en una universidad que sigue un modelo profesionalizante.

\footnotetext{
Es trabajo forma parte de la tesis doctoral titulada "Configuración de comunidades académicas en la Universidad Nacional Autónoma de Honduras. El caso de Psicología, Medicina y Economía", desarrollada en el programa de Doctorado en Educación de la Universidad Pedagógica Nacional Francisco Morazán. La autora agradece los aportes que han brindado en distintos momentos el Dr. Oscar Soriano y el Dr. German Moncada.

* Docente del Departamento de Ciencias de la Educación, Universidad Pedagógica Nacional Francisco Morazán

Recibido 29 de septiembre 2017 / Aceptado 31 de octubre 2017.
}

INIEES - UPNFM 
Una propuesta analítica para el estudio de las comunidades académicas en el contexto de la institución universitaria

Palabras Clave: comunidad académica, perspectiva normativa, perspectiva interpretativa, componentes estructurales, universidad, trabajo académico, libertad académica, ethos académico y cultura académica

\begin{abstract}
The aim of this paper is to present an analytical framework for the study of the academic community in the context of the university. Integrating two approaches, one policy where defined, from the theoretical and empirical review, the structural components of a community, such as: academic work, academic freedom, academic ethos and academic culture. Moreover, from an interpre-

tative approach interested to analyze how members of a community build their understanding of the defined structural components. Finally, from the articulation of the two approaches, it seeks a comprehensive understanding of how the structural components are configured in an academic community, which is inserted into a university that follows a professionalizing model.
\end{abstract}

Keywords: academic community, normative perspective, interpretative perspective, structural components, university, academic work, academic freedom, academic ethos and academic culture.

\title{
Introducción
}

El estudio de las comunidades académicas es un tema de relevancia en la actualidad, por su responsabilidad en el cultivo de la ciencia, así como en la producción y validación de conocimiento a través del desarrollo de la investigación científica. Lo que se entiende por comunidad académica es una conceptualización compartida a nivel internacional, de ahí que la riqueza conceptual y metodológica se encuentra en las perspectivas desde donde se ha estudiado. Así pueden 
identificarse trabajos en Antropología de la Ciencia (Becher, 2001), Sociología de la Ciencia (Bourdieu, 2003); Sociología de la Educación Superior (Clark, 1987,1997) e Historia de la Ciencia (Kuhn $(1971,1978)$, entre otros.

En este trabajo se propone una estructura analítica que articula dos perspectivas: una normativa y otra interpretativa de la ciencia. Este es así ya el objetivo es estudiar la presencia o ausencia de los rasgos distintivos de comunidades académicas en el contexto de la institución universitaria, que sigue un modelo de universidad profesionalizante y no un modelo de investigación, desde donde se ha hecho la mayoría de estos estudios.

La perspectiva "normativa" o "interpretativa" de la ciencia, suponen dos formas de análisis diferentes respecto al trabajo científico que se realiza dentro de una comunidad. Desde una perspectiva normativa se discute fundamentalmente sobre el ser y deber ser de esta particular formar de agrupación. El precursor de esta perspectiva fue Robert Merton (2002) quien en 1940, definió cuatro normas que deben regular el trabajo científico, siendo estas: el universalismo, comunismo, desinterés y escepticismo organizado.

En la perspectiva interpretativa, el objetivo se centra en comprender cómo las normas que rigen el trabajo científico son interpretadas al interno de una comunidad, y los factores externos que pueden influir, ya que se considera que el "aprendizaje científico debe ser tratado como un proceso activo de interacción y no como una situación en la que el novicio absorbe estructuras normativas pasivamente" (Law, \& French, 1994, p. 54). Este proceso de interpretación se construiría a través de la intersubjetividad, la cual se concibe como el "intento normativo de cada uno respecto de todos" (Fagot-Lagueault, 2011, p. 150).

Ambas perspectivas han sido vistas como irreconciliables, sin embargo, en este trabajo, se propone su articulación, ya que interesa estudiar la comunidad académica desde una visión normativa, y a partir de la 
Una propuesta analítica para el estudio de las comunidades académicas en el contexto de la institución universitaria

visión interpretativa comprender cómo la normatividad científica cobra vida al ser intersubjetivamente construida en una comunidad, ya que "el trabajo de quien estudia a los académicos y sus comunidades no es decir (...) como deben comportarse, ni estigmatizar sus malas conductas. Lo que interesa es comprender el significado de la separación entre las normas y las prácticas" (Fagot-Lagueault, 2011, p. 150).

\section{Discusión Teórica}

$\bullet \bullet \bullet \bullet \bullet \bullet \bullet \bullet \bullet \bullet \bullet \bullet \bullet \bullet \bullet \bullet \bullet \bullet \bullet \bullet \bullet \bullet \bullet \bullet \bullet \bullet \bullet \bullet \bullet \bullet \bullet \bullet \bullet \bullet \bullet \bullet \bullet \bullet \bullet \bullet \bullet \bullet$

Las comunidades académicas desde una perspectiva normativa

¿Qué es una comunidad académica? Es un tipo particular de grupo, organizado en torno a la dinámica de producción de conocimiento, y donde el trabajo científico y sus productos, son regulados por normas aceptadas por todos. Normativamente, se emplea el concepto en singular, ya que permite distinguir esta forma particular de organización, de otras formas de comunidad que puedan existir dentro de la universidad o en la sociedad. Cuando se analiza el concepto en plural, alude a las diferencias que se pueden encontrar entre comunidades como resultado del análisis empírico y que está dado por el tipo de disciplinas científicas o especialidades que cada comunidad cultiva las marcadas diferencias en las tareas cognitivas y operativas que desarrollan (ver, Becher, 2001).

En esencia, una comunidad académica es aquella que cultiva la ciencia y tiene como tarea esencial la investigación (Casas, 1980; Becher, 2001; Bourdieu, 2003; Kunh, 1971; Pedró, 2002), por ello puede existir fuera de la institución universitaria, ya que se centra fundamentalmente en el cultivo de la ciencia o de una disciplina científica (Clark, 1987, 1997). En este tipo de comunidades los vínculos y relaciones se producen a través de redes nacionales e internacionales, siendo reconocidas como "comunidades científicas" (Casas, 1980; Kunh, 1971). 
Una comunidad académica, si bien pueden ser comunidad científica, se distinguen porque además de producir y validar conocimiento a través de la investigación científica, responde a otros objetivos más amplios como la formación de profesionales y científicos, así como la promoción del saber y la cultura, entre otros, ya que está inserta en la institución universitaria.

La comunidad académica se analiza en el contexto de la institución universidad, pues según Clark, (1997) con el surgimiento en Alemania, de la universidad moderna a fines del siglo XIX y principios del S XX, se considera como rasgo distintivo de una universidad el cultivo de la ciencia a través de la investigación científica. Este rasgo se mantiene hasta la actualidad a través del "modelo de universidad de investigación" (Salmi, 2009) y que es reflejado a través de los diferentes rankings mundiales de universidades.

Dos exponentes de la perspectiva normativa en la ciencia son Robert Merton y Thomas Kuhn. Merton (2002), al analizar la dinámica de las personas que se dedican al trabajo de la ciencia, estableció el concepto de ethos de la ciencia, como "un complejo de valores y normas efectivamente templados que se consideran obligatorios para el hombre de ciencia" (p. 637); está inserto en un modo de vida que se refleja en los siguientes elementos constituyentes "universalismo, comunismo, desinterés, y escepticismo organizado" (Merton, 2002, pp. 639-346).

Para Kuhn (1971) una comunidad fundamenta su acción en base a un "paradigma", concebido como un "modelo o patrón aceptado" (Kuhn, 1971). Un paradigma es aquello que una "comunidad científica, y sólo ellos comparten. La posesión de un paradigma constituye un grupo de personas en una comunidad científica, grupo que de otro modo estaría formado por miembros inconexos" (Kunh, 1971:13). Posteriormente, ante las contradicciones que generó el concepto de paradigma, Kuhn cambia el concepto "matriz disciplinar" (1978).

Al establecer un marco normativo, para el desarrollo trabajo científico dentro de una comunidad, se "asume un alto grado de consenso cognitivo y normativo, y busca como uno de sus primeros objetivos 
Una propuesta analítica para el estudio de las comunidades académicas en el contexto de la institución universitaria

identificar un conjunto relativamente estable de normas, al que se considera simultáneamente, la guía del científico en su trabajo y de los rasgos definitorios de la naturaleza institucional de la ciencia" (Law \& French, 1994: 53).

La comunidad académica desde una perspectiva interpretativa

Desde una perspectiva interpretativa, interesa la comprender cómo las normas se reflejan en las prácticas y discursos científicos en un contexto determinado. Se asume, que la configuración de una comunidad académica es un proceso constructivo que indica diferentes grados de madurez en el cultivo de la ciencia y la investigación científica, siendo este un proceso colectivo, no individual.

Posibilita el análisis en las comunidades donde todavía no se ha alcanzado un alto consenso cognitivo y normativo, a fin de comprender "el significado de la separación entre las normas y las prácticas" (FagotLargueault, 2011, p. 150), o distinguir entre "acción científica y no científica" (Law \& French, 1994, p. 54), así como la relación o influencia que puedan existir respecto a factores externos. Desde la perspectiva normativa, los factores externos no tendrían relevancia para el análisis ya que las normas son compartidas por todos, pero al no existir alto consenso cognitivo y normativo dentro de un grupo, este análisis se hace relevante, ya que permite comprender la configuración de una comunidad académica y del trabajo científico, no como producto, sino como un proceso que puede ser influido por diferentes factores internos y externos.

La articulación de la perspectiva normativa e interpretativa: una propuesta analítica

A partir de la articulación de las dos perspectivas planteadas, se desarrollan los componentes estructurales en el contexto de una universidad con modelo profesionalizante. En la figura 1, se identifican los conceptos centrales de la estructura analítica propuesta, a partir de la perspectiva normativa se definen los componentes estructurales 
que serán objeto de estudio en una comunidad, posteriormente desde la perspectiva interpretativa se analiza la distancia existente entre la norma y la práctica. En resultado de este análisis se define como configuración, la cual es definida como una estructura constitutiva, que refleja una relación comprehensiva entre los diferentes componentes estructurales. Permite la identificación de configuraciones particulares entre las comunidades, así como sus rasgos comunes, que expresan a su vez, una relación con el contexto institucional, con el desarrollo de la ciencia, el trabajo científico, y el vínculo entre investigación, docencia y aprendizaje. El análisis de las configuraciones se realiza desde la perspectiva de los académicos, ya que interesa comprender cómo los marcos normativos cobran vida en contextos específicos. Se asume que las configuraciones son dinámicas, y que las mismas reflejan los niveles de madurez académica alcanzados por una comunidad en un momento determinado. Esto es así porque:

Más que una comunidad racional, la comunidad científica (académica ${ }^{9}$ ) es la que tiene la exigencia de racionalidad. Tal exigencia se traduce en aquella formulación de reglas metodológicas y deontológicas (éticas) que pueden evolucionar y ser transgredidas. La normatividad viva que está en el funcionamiento de dentro de una comunidad no es "trascendental" (...) es un hecho observable que puede ser el objeto de estudios históricos, sociológicos; también le interesa a la Filosofía de las Ciencias (Fagot-Lagueault, 2011, p. 150).

\footnotetext{
9 Agregado por la autora de este artículo.
} 
Una propuesta analítica para el estudio de las comunidades académicas en el contexto de la institución universitaria

Figura 1

Propuesta analítica para el estudio de las comunidades académicas en el contexto de I instituciónuniversitaria ${ }^{10}$

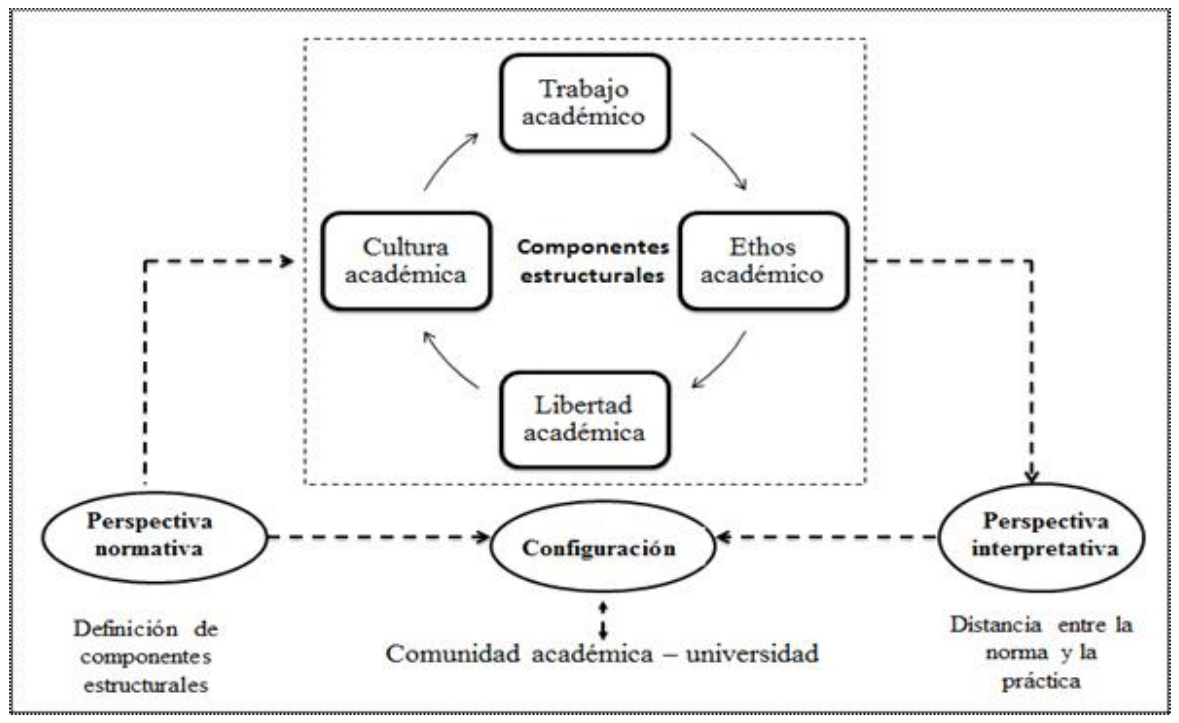

A partir de la revisión teórica y empírica se identificaron cuatro componentes estructurales de una comunidad académica (ver figura 1), lo cual refleja un consenso normativo, estos son: trabajo académico (Barnett, 2008; Becher, 2001; Boyer, 1999; Clark, 1997), ethos académico, (Merton, 2002; Albornoz, 2007, Dill, 2008), libertad académica (Albornoz, 2009; Nelson, 2010; Soriano, 2008), cultura académica (Clark, 1980; Valima, 2008; Sporn, 1996; Gines Mora, 2001). Los componentes estructurales propuestos se definen a continuación.

El trabajo académico explica las distintas dimensiones y espacios del trabajo que se realiza dentro de una comunidad, refleja una estructura cognitiva y operativa que está guiada por la disciplina científica o especialidad que trabajan. Boyer (1999), señala que el trabajo académico debe ser comprendido, no desde las "categorías rígidas de investigación, enseñanza y servicio" (p.35), sino desde una comprensión más flexible y dinámica de las funciones, como ser:

${ }_{10}$ A partir de la revisión teórica y empírica, esta propuesta analítica es realizada por la autora del artículo.

<Paradigma> - Revista de Investigación Educativa. Año 24. No. 38 
"trabajo académico de descubrimiento, trabajo académico de interpretación, trabajo académico de aplicación y trabajo académico de enseñanza". Por su parte, Barnett (2008) propone que el estudio de la universidad en la actualidad debe comprenderse desde tres espacios: Espacio pedagógico y curricular, espacio del saber y espacio intelectual. Desde esta perspectiva la universidad se concibe con una "arquitectura propia en donde el trabajo académico adopta formas que configuran patrones sujetos a cambios constantes" (Barnett, 2008:15). Esta comprensión del trabajo académico permite un análisis de las diferencias que se presentan entre instituciones y dentro de las instituciones. Para Clark (1999)

(...) el trabajo académico, se organiza en torno al conocimiento, por ello, las actividades académicas por sus rasgos específicos modelan las organizaciones y crean problemas particulares de comportamiento y de poder. (...) El trabajo académico es específico ya que se construye sobre la base de esfuerzos tendientes a descubrimiento, la conservación, la depuración, la transmisión y la aplicación. El material es el conocimiento, y las tecnologías son la investigación y la enseñanza.

Respecto al ethos académico, en este trabajo se distinguen tipos: el ethos de la ciencia y el ethos institucional. El ethos de la ciencia según Merton, (2002, pp. 639-346) se rige por cuatro principios: "universalismo, comunismo, el desinterés y el escepticismo organizado". Los cuales pueden verse debilitados por prácticas y conductas contrarias a su cultivo, así lo señala Bunge (2001, p.312) al identificar conductas pseudocientíficas y las anticientíficas

Los anticientíficos que con frecuencia se llaman postmodernos enseñan que no hay verdades ni universales ni objetivas [...]. Por su parte los seudocientíficos introducen de contrabando conceptos borrosos, conjeturas extravagantes o incluso ideología como si fueran conceptos científicos.

Lo anterior refleja que una comunidad académica con un ethos de la ciencia fuerte esta vigilante al cumplimiento de las normas y principios 
Una propuesta analítica para el estudio de las comunidades académicas en el contexto de la institución universitaria

científicos y a descubrir aquellos discursos y prácticas que pueden debilitarlo. Debe destacarse que en una institución universitaria pueden existir comunidades académicas con diferentes niveles de fortaleza en su ethos científico, y como se discutirá más adelante la misma institución puede influir con su dinámica y cultura organizativa a su debilitamiento, sobre todo cuando donde existen altos niveles de autocomplacencia institucional, porque el trabajo académico no responde a estándares científicos, sino a otro tipo de criterios como ser políticos y económicos, o de determinados grupos de poder.

El ethos institucional, hace referencia a que la universidad es una institución regulada por un conjunto de normas cuyo objetivo es promover las tres funciones de la universidad moderna: docencia, investigación y vinculación con la sociedad. En este marco normativo la universidad define unidades encargadas de venta de servicios, para las cuales designa investigadores quienes tienen salario de la universidad, pero además devengan su salario de los proyectos que coordinan. Estos proyectos se caracterizan por aportar a la universidad recursos económicos, patentes, los cuales consolidan el capital científico, intelectual y cultural de estas instituciones. Estas son prácticas institucionalizadas a través de marcos jurídicos, financieros y éticos que promueven y exigen la rendición de cuentas de parte de estos equipos de investigadores, lo que se traduce en una gestión institucional transparente. Permite el desarrollo del capital intelectual a través de redes institucionales, nacionales e internacionales, por tanto, hay una valoración positiva de la vinculación con otras instituciones y equipos con estándares similares.

Sin embargo, se están produciendo dos dinámicas dentro de las universidades que lleva al debilitamiento de la conducta académica de sus profesores respecto al marco ético del desarrollo de la investigación y la docencia, debido a las creencias instaladas sobre las mismas y los marcos que las regulan, o través de un debilitamiento de los marcos epistémicos, producto de la instalación de un pensamiento único dentro de la universidad como forma de control del trabajo académico, produciendo prácticas de populismo y autoritarismo. 
Actualmente entre el vínculo docencia e investigación se están produciendo una serie de tensiones y contradicciones que debilitan el trabajo académico. Esto se debe a las presiones externas y a las creencias que se han instalado respecto que lo que se considera valioso como trabajo académico. Actualmente los discursos, enfatizan en la urgencia de desarrollar investigación de alto nivel, sin considerar, las capacidades de las instituciones universitarias para ello, y además de ignorar o desentenderse de una serie de conductas académicas que se presentan dentro la universidad y que afectan el trabajo académico.

Dill (2008) señala que estas son prácticas "degradación de la ética académica", como producto del debilitamiento de los marcos de "regulación profesional". Se ha encontrado que los profesores cultivan prácticas académicas que están en contra de la visión de la universidad como bien público bajo el amparo de la libertad y autonomía académica. Dill (2008) señala que, si los profesores "desean insitir en la necesidad de autonomia académica, deben ofrecer pruebas irrefutables, tanto de cara a comunidad como al público en general, de que los procesos colegiados para el mantenimiento de los niveles académicos son firmes y válidos" (p. 232).

Otro tipo de practicas contrarias al ethos de la ciencia e insitucional, son señaldas por Albornoz (2007) y Bartra (2001), siendo estas el populismo académico y la mentalidad autoritaria. Se relacionan con la libertad académica, cuando las autoridades y docentes de una insitucion no establecen limites para su ejercicio. El populismo académico ha sido estudiado como un tipo de práctica que se cultiva en instituciones universitarias, y sus niveles de presencia dependerá de la madurez alcanzada en el ethos académico, para poder revertir las influencias externas que se instalan a lo interno de las universidades (Albornoz, 2008, Bartra, 2001). Lo anterior, se basa en la premisa de que "ninguna universidad es una isla" (Nelson, 2011), al ser instituciones que "forman parte de la sociedad" (Barnett, 2001), las universidades son instituciones permeables a su contexto y a sus influencias. Esta permeabilidad al contexto, en el caso latinoamericano se da en un marco en donde las universidades son consideradas 
Una propuesta analítica para el estudio de las comunidades académicas en el contexto de la institución universitaria

"constructoras de Estado" (Ondorika, 2011), ya que siempre han tenido un rol protagónico en el desarrollo de las políticas y proyectos de Estado, y esta relación ya sea para bien o para mal, tiene una enorme influencia en la vida universitaria.

No es extraño que, así como en la sociedad se pueda calificar a la política partidaria como populista, este mismo tipo de expresiones se empleen para explicar una serie de prácticas que han permeado en diferentes niveles a las instituciones universitarias. Es así como el populismo académico, tiene diferentes formas de manifestación y se ha naturalizado como un estilo de vida que forma parte de la universidad, pues "si no se dispone de universidades capaces de ser fuentes permanentes de creación de nuevos conocimientos, se estará en incapacidad de interceptar mapas mentales que dominan el mundo contemporáneo" y la tendencia será a atarse de forma irreflexiva a tendencias y prácticas ya superadas" (Albornoz, 2009, p. 227). Este retroceso en la generación de nuevas formas de interpretar el mundo es producto del debilitamiento del ethos de la ciencia y la universidad pasa de ser una institución que cultiva el pensamiento plural, de "no tener condición" (Derridá, 1960), a una institución "condicionada, regulada" (Albornoz, 2009). En este contexto aparece la mentalidad autoritaria que "es propia de las formas de control institucional y del pensamiento. Es la negación del principio de negociación, que es a su vez propio de la mentalidad democrática" (Albornoz, 2007 p. 41).

En contextos donde existe una libertad académica y ethos académico fuerte, orientado al cultivo de la ciencia y al ser/deber ser de la universidad, existen instancias normativas definidas que orientan y regulan el quehacer universitario, el cual se ejerce a través de una dinámica de pesos y contrapesos en donde las comunidades académicas y sus académicos, son exigidas a asumir su rol en el desarrollo de la ciencia y de las actividades de investigación. Pero, además, como las comunidades académicas trascienden las fronteras institucionales, la vigilancia se ejerce desde otras comunidades manifestándose a través del cuestionamiento de conductas científicas impropias, a través de las mismas publicaciones reconocidas por el 
mundo científico, lo que también es aplicable a las instituciones universitarias.

El componente de libertad académica, para Nelson (2010), encarna los compromisos en la búsqueda de conocimiento; Albornoz (2007: 166-167) refiere al "derecho para enseñar y discutir, hacer investigación y publicar sus resultados, expresar una opinión sin intimidaciones y presiones, no es análoga a conductas impropias o inmorales, y no está diseñada para proteger la ineptitud e improductividad de los académicos". Por su parte, Soriano (2008) identifica tres perspectivas desde donde se justifica la libertad académica y estas son "epistemológicas, políticas y morales", de las tres la perspectiva epistemológica es fundamental, ya que se asume la verdad y el conocimiento como un proceso que debe ser construido y ajustado permanentemente.

No debe entenderse la libertad académica como ausencia de límites, los cuales deben ser establecidos y regulados al interior de la comunidad académica, por pares académicos de otras comunidades, y por marcos normativos institucionales. Es así como la libertad académica no existe en el vacío, ya que se articula a nivel universitario con la autonomía universitaria, y a nivel de la formación y el quehacer académico con el ethos académico. Albacth (2009, p. 1), por su parte, sostiene que "todo mundo parece estar en favor de la libertad académica (...). Sin embargo, existen problemas prácticamente en todas partes - creados por las cambiantes realidades académicas, presiones políticas, la creciente comercialización y marketización de la educación superior o presiones legales".

La cultura académica, es un componente multidimensional, ya que estaría referido según Clark (1980) a la cultura de la "disciplina, profesión, institución y sistema". Se estaría hablando que diferentes tipos de cultura relacionadas entre sí, que expresan niveles de consolidación. Las diferentes dimensiones de la cultura institucional pueden verse condicionadas cuando desde lo externo para Välimaa (2008), desde una "perspectiva neoliberal se enfatiza el rol de las 
Una propuesta analítica para el estudio de las comunidades académicas en el contexto de la institución universitaria

universidades como cruciales en la producción de conocimiento evidenciando una comprensión industrial" y cuando la dinámica institucional produce un debilitamiento de la cultura académica centrada en el trabajo científico, presentándose un patrón de "endurecimiento burocrático y ablandamiento académico" (Padrón, 2005). Este patrón explica que en las instituciones los estándares académicos y científicos que se construyen de forma colegiada son sustituidos por perspectivas individualistas y subjetivistas, y las dinámicas organizativas y administrativas son altamente burocratizadas, prevalecen los reglamentos, las relaciones verticales y mecanizadas (Padrón, 2005). En este contexto la cultura académica puede sufrir un proceso de fragmentación que es paulatino y que va desdibujando a las comunidades académicas y las universidades.

\section{Conclusiones}

Con esta propuesta analítica se pretende articular las perspectivas normativa e interpretativa de la ciencia, a fin de estudiar los rasgos que asumen las comunidades académicas que están insertas en universidades profesionalizantes. Interesa comprender la configuración que asumen los diferentes componentes estructurales e identificar las condiciones institucionales que pueden influir en la misma.

Como puede apreciarse, los diferentes componentes están relacionados entre sí y el debilitamiento en uno de ellos afecta al otro, es así como trabajo que se realiza en las comunidades académicas refleja una estructura comprensiva que está relacionada con la institución universitaria. 


\section{Referencias Bibliográficas}

Albornoz, O. (2009). Breves notas sobre autonomía y la libertad académica. Caracas: EBUC.

Albornoz, O. (2007). La libertad académica y la mentalidad autoritaria. Caracas: EBUC.

Altbach, P. (2009). La libertad académica amenazada. Campus Milenio, Núm. 337. Seminario de Educación Superior, México: UNAM. Recuperado de: http://www.ses.unam.mx/publicaciones/ articulos. php?proceso=visualiza\&idart $=703$

Barnett, R. (2001). Los límites de la competencia. Barcelona: Gedisa.

Barnett, R. (2008). Para una transformación de la universidad. Barcelona, España: Editorial Octaedro.

Bartra, R. (2001). El fin del populismo académico autoritario. Revista Pensamiento Educativo, Año 9, abril, 12-17.

Becher, T. (2001a). Tribus y territorios académicos. 1ra edición. Barcelona, España: Editorial Gedisa.

Bourdieu, P. (2003). El oficio del científico. Barcelona, España: Editorial Anagrama.

Boyer, E. (2003). Una propuesta para la educación superior del futuro. 2da. Reimpresión. México, D.F.: Fondo de cultura Económica.

Casas Guerrero, R. (1980). La idea de comunidad científica: su significado teórico y su contenido ideológico. Revista Mexicana de Sociología, 42 (3) 1217-1230.Recuperado de http://www.jstor.org/ stable/3539999. 
Una propuesta analítica para el estudio de las comunidades académicas en el contexto de la institución universitaria

Clark, B. (1980). Academic Culture. Higher Education Research Group. Pp. 4-33. New Haven: Yale University.

Clark, B. (1987). The higher education system. California: University California Press.

Clark, B. (1997). Las universidades modernas como espacios de investigación y docencia. México: Miguel Ángel Porrúa.

Dill, D. (2008). La degradación de la ética académica: Docencia, investigación y renovación de la autorregulación profesional. En R. Barnett (Ed.), Para una Transformación de la Univerisdad. Pp. 231-246. Madrid:Octaedro.

Fagot-Lagueault, A. (2011). La construcción intersubjetiva de la objetividad científica. En D. Andler, A. Fagot-Largeault y B. Saint-Sernin (Coord.), Filosofía de las Ciencias. México: Fondo de Cultura Económica.

Kuhn, T. (1982). La tensión esencial. México: Fondo de cultura Económica.

Kuhn, T. (1978). Segundos pensamientos sobre paradigmas. Madrid: Tecnos.

Kuhn, T. (1971). La estructura de las revoluciones científicas. 1ra edición en español. México D.F., México: Fondo de Cultura Económica.

Merton, R. K. (2002). Teoría y estructuras sociales. México: Fondo de Cultura Económica.

Mora, J-G. (2001). Governance and management in the new university. Tertiary Education and Management, 7, 95-100.

Nelson, C. (2010). No University is an Island: Saving Academic Freedom. New York: New York U Press. 
Law, J. \& French, D. (1994). Sociologías normativa e interpretativa de la ciencia. En J.M. Iranzo, J.R. Blanco, T. González de la Fe, C. Torres \& A. Cotillo (Coord.). Sociología de la ciencia y la tecnología. España: CSIC

Padrón, J. (2005): Los Doctorados en Ciencias Sociales: Ablandamiento académico y endurecimiento burocrático. Informe de Investigaciones Educativas. Vol. 19. Recuperado de http://biblo.una.edu.ve/ojs/ index.php/IIE/search/titles?searchPage $=5$. Consultado el jueves 22 de enero de 2010.

Pedró, F. (2004). Fauna Académica. Barcelona: UOC.

Salmi, J. (2009). El desafío de crear universidades de rango mundial. Washington, D.C.: Banco Mundial

Soriano, O. (2008). Los estudios de postgrado: Libertad académica y líneas de investigación. En O. Soriano (Coord. y Comp.), Problemas Educativos en la Sociedad Contemporánea. pp. 9-28 Tegucigalpa: UPNFM.

Sporn, B. (1996). Managing university culture: an analysis of the relationship between institutional culture and management approaches. Higher Education, 32, 41-61.

Välimaa, J. (2008). Cultural studies in higher education research. En J. V. Ylijoki and O.H. Ylioki (Eds.), Cultural Perspective on Higher Education. Pp. 9-25. Finland: Springler. 